\title{
867 TRITAC-XR IS AN EXTENDED-RELEASE T CELL ENGAGER PLATFORM DESIGNED TO MINIMIZE CYTOKINE RELEASE SYNDROME BY REDUCING CMAX IN SYSTEMIC CIRCULATION
}

Kathryn Kwant*, Sony Rocha, Katrina Stephenson, Maria Dayao, Subramanian Thothathri, Rose Banzon, Wade Aaron, Golzar Hemmati, Evan Callihan, Timothy Yu, Jessica O'Rear, Eric Bragg, Willis Kwong, Hubert Situ, Avneel Hundal, Stephen Yu, Taggra Jackson, Kevin Wright, Yinghua Xiao, Linh To, Richard Austin, Bryan Lemon, Holger Wesche, S Jack Lin. Harpoon Therapeutics, South San Francisco, CA, USA

Background CD3-targeted $\mathrm{T}$ cell engagers are potent antitumor therapies, but their development often requires management of cytokine release syndrome (CRS). Subcutaneous dosing is a promising strategy to reduce CRS, but its application is limited by its increased immunogenicity risks. Subcutaneous dosing is hypothesized to mitigate CRS by reducing the maximum drug concentration (Cmax) and preserve efficacy by maintaining the same minimum drug concentration (Cmin) as intravenous dosing. A $\mathrm{T}$ cell engager designed to be dosed intravenously but engineered to mimic the PK properties of subcutaneous dosing could alleviate CRS without increasing immunogenicity.

Methods TriTAC-XR molecules are engineered $\mathrm{T}$ cell engager prodrugs that become slowly activated in systemic circulation. This extended-release mechanism results in a slow build-up of circulating active drug, similar to subcutaneous dosing, and extends drug exposure to enable longer dosing intervals. The prodrug was engineered by adding a peptide mask and protease-cleavable linker to the N-terminus of a TriTAC, a constitutively active and half-life extended $\mathrm{T}$ cell engager. The mask binds to the anti-CD $3 \epsilon$ domain and prevents $\mathrm{T}$ cell binding. Upon cleavage by systemic proteases, active $\mathrm{T}$ cell engager is released. Binding was assessed using ELISA on recombinant $\mathrm{CD} 3 \epsilon$ protein and using flow cytometry on primary $\mathrm{T}$ cells. $\mathrm{T}$ cell engager function was assessed using $\mathrm{T}$ cell-dependent cellular cytotoxicity (TDCC) assays with resting human $\mathrm{T}$ cells. Safety and efficacy were modeled in non-human primates.

Results TriTAC-XR had markedly reduced binding to recombinant $\mathrm{CD} 3 \epsilon$ protein and to primary $\mathrm{T}$ cells as well as reduced potency in functional TDCC assays compared to its unmasked active drug. In cynomolgus monkeys, TriTAC-XR had significantly attenuated cytokine production while maintaining comparable pharmacodynamic effects as a non-masked active drug. The ratio of Cmax to Cmin for the active TriTAC-XR was significantly smaller than a non-masked control.

Conclusions TriTAC-XR is activated in a time released manner by systemic proteases to minimize differences between the Cmax and Cmin of systemic active drug. This mechanism is different from other protease-activated $\mathrm{T}$ cell engager prodrugs that are only activated by tumor-associated proteases. Compared to canonical $\mathrm{T}$ cell engagers, TriTAC-XR is expected to improve safety by reducing CRS and to provide convenience by extending dosing intervals.

http://dx.doi.org/10.1136/jitc-2021-SITC2021.867 